\title{
Tuberculosis Caused by Mycobacterium tuberculosis Complex in a Captive Tapir (Tapirus terrestris)
}

\author{
Washington Luiz Assunção Pereira ${ }^{1}$, Marcella Katheryne Marques Bernal ${ }^{1,6}$, Carlos Eduardo Dias Campos ${ }^{2}$, \\ José de Arimatéia Freitas ${ }^{3}$, Yan Corrêa Rodrigues ${ }^{4}$, Karla Valéria Batista Lima ${ }^{4,5}$, \\ Amanda Desirée Assunção Cecim ${ }^{5} \&$ Alex Júnior Souza de Souza ${ }^{5}$
}

\begin{abstract}
Background: Tuberculosis is an infectious disease caused by the Mycobacterium tuberculosis complex has already been identified in a great number of wild species. Captive animals may have a potential source for zoonoses, because is related to factors such as cohabitation, direct contact with the public, presence of biological vectors, nutritional deficiencies, absence of sanitary barriers, precautionary hygienic measures and sanitary vigilance. In Brazil, there is little information on tuberculosis in captivity animals, and little attention is given to the risks of disease transmission from humans. The aim of this study was to report the first diagnosed case of tuberculosis Tapirus terrestris in Brazilian Amazon region.

Case: One Tapirus terrestris was kept by local zoobotanic foundation in city of Marabá, Southeast of Pará state, Brazilian Amazon, and became ill. Physical examination revealed cough, sneezing, nasal outflow, dyspnea, hyperthermia and lethargy, leading to death. Necropsy demonstrated severe pulmonary alterations: thickening of the inter-alveolar septa, alveolar emphysema, and miliary nodules with dimensions up to $5 \mathrm{~mm}$, which were yellowish-white, caseous, and sometimes calcified. Additionally, large areas of caseous compaction of the parenchyma, characteristic of caseous tuberculosis. Histopathological analysis revealed a process characteristic of mycobacterial infection, with alveoli filled with caseous exudate and thickened septa and fibrocytes, in addition to recently formed tubercles, some with caseous necrosis, calcifications and Langhans cells. In the Ziehl-Neelsen staining, alcohol-acid resistant bacilli were observed in the mesenteric lymph nodes. No mycobacterial growth was observed in Lowenstein-Jensen culture medium. A nested PCR followed by a sequencing assay targeting the $h s p 65$ gene and M. tuberculosis complex member was detected. Water and M. tuberculosis $\mathrm{H} 37 \mathrm{Rv}$ were used as negative and positive controls, respectively.

Discussion: This article reports the first case of tuberculosis in T. terrestris in Amazonia. The case of infection was caused by a complex of Mycobacterium tuberculosis, as well as reports of other studies described in wild animals. This record presented clinical and pathological similarity with the cases of a tapir and coati at the zoo of Curitiba, and non-human primates in bioteries in Belém. The disease was reported, also, in a T. indicus female in Bangkok, Thailand. In another study with two tapirs there was positivity of tuberculin test result in a Swedish zoo. In necropsy findings of tuberculosis in a Malayan tapir showed multiple white, caseous nodules spread throughout the lungs. Studies have shown that lesions can be in various organs, among them the mediastinal and mesenteric lymph nodes, liver, spleen, kidneys, ovaries, serosa, diaphragm, intestines and uterus. For exemple in a case of disseminated tuberculosis caused by M. tuberculosis in a Malayan tapir were described multiple granulomes, some coalescent, characterized by necrotic caseous centers, with some Langhans giant cells and a discrete fibrotic zone. In the present work, the results of the anatomopathological and molecular assays confirmed the clinical suspicion of respiratory infection and established the conclusive diagnosis of pulmonary tuberculosis caused by $M$. tuberculosis complex member. It is worth noting that tuberculosis is an important zoonotic disease affecting survival of the species, posing an extinction threat and also a public health concern.
\end{abstract}

Keywords: Mycobacterium tuberculosis, Tapirus terrestris, tuberculosis, captivity, zoonosis. 


\section{INTRODUCTION}

Tuberculosis is an infectious bacterial disease caused by Mycobacterium tuberculosis complex [1]. The disease has already been identified in a great number of wild species, including non-human primates, sheep and other exotic ungulates, carnivores, sea mammals and parrots $[2,4,6,7]$.

Buildings designed for animal captivity are environments with an elevated risk of occurrence for a wide range of diseases, including zoonosis. The animals inhabiting these places are originally from different geographic areas and are kept for sufficient time to contract or transmit diseases to humans [3].

The occurrence of tuberculosis in wild animals under natural conditions and in captivity is related to factors such as cohabitation, direct contact with the public, presence of biological vectors, nutritional deficiencies, absence of sanitary barriers, precautionary hygienic measures and sanitary vigilance $[3,6]$. From an epidemiologic point of view, it is important to assess the risks represented by potential sources of infection present in captivity, for both humans (keepers, veterinarians, biologists and visitors) and animals. In Brazil, there is little information on tuberculosis in captivity animals, and little attention is given to the risks of disease transmission from humans to domestic and wild animals and from these animals to humans. The aim of this study was to report the first diagnosed case of tuberculosis Tapirus terrestris in Brazilian Amazon region.

\section{CASE}

One captive tapir (Tapirus terrestris) was kept by local Zoobotanic Foundation (Zoobotanical Foundation of Marabá) in city of Marabá, Southeast of Pará state, Brazilian Amazon region, and became ill. Physical examination revealed cough, sneezing, nasal outflow, dyspnea, hyperthermia and lethargy, leading to death. Necropsy demonstrated severe pulmonary alterations: thickening of the inter-alveolar septa, alveolar emphysema, and miliary nodules with dimensions up to $5 \mathrm{~mm}$, which were yellowish-white, caseous, and sometimes calcified. Additionally, large areas of caseous compaction of the parenchyma, characteristic of caseous tuberculosis, were observed (Figure 1).

Histopathological analysis revealed a process characteristic of mycobacterial infection, with alveoli filled with caseous exudate and thickened septa and

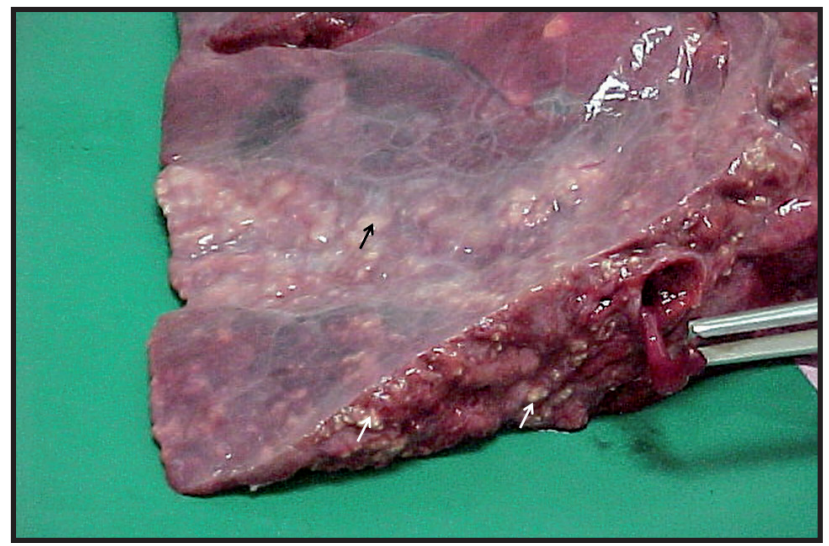

Figure 1. Lung, tapir (Tapirus terrestris). A Segment of the lung lobe presenting numerous tiny whitish nodules with an irregular distribution (arrows).

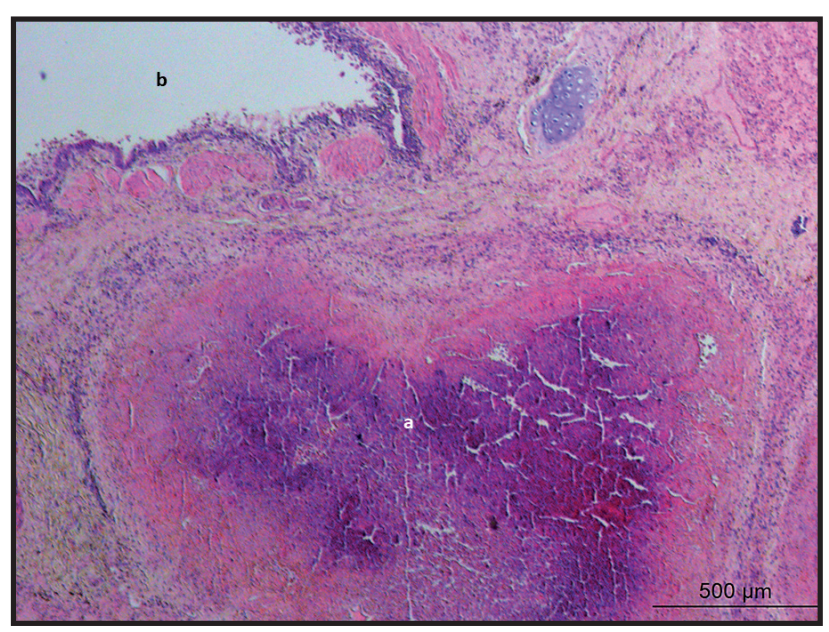

Figure 2. Photomicrograph showing alveoli filled with exudative and necrotic debris, fibrosis of the septa with parenchymal extension and the presence of macrophages and giant Langhans cells. [HE, 20x magnification]. Lung (Tapirus terrestris).

fibrocytes, in addition to recently formed tubercles, some with caseous necrosis, calcifications and Langhans cells (Figure 2). In the Ziehl-Neelsen staining, alcohol-acid resistant bacilli were observed in the mesenteric lymph nodes. No mycobacterial growth was observed in Lowenstein-Jensen culture medium.

A nested PCR followed by a sequencing assay targeting the $h s p 65$ gene was carried out as previously described by Telenti et al. [10] and M. tuberculosis complex member was detected. Water and $M$. tuberculosis $\mathrm{H} 37 \mathrm{Rv}$ were used as negative and positive controls, respectively.

\section{DISCUSSION}

This article reports the first, clinically, anatomopathologically, microbiologically and molecular diagnosed case of tuberculosis in Tapirus terrestris in Amazonia. The case presented herein showed 
clinical and anatomopathological similarities with the infections of a tapir and coati at the zoo of Curitiba, Paraná state [8], and non-human primates in bioteries in Belém, Pará state [4]. In the present case, as in the others previously reported, Mycobacterium tuberculosis complex was detected identified.

The disease was reported in a tapir in Bangkok, Thailand, occurring in a 17-year-old female Malayan tapir (Tapirus indicus) [5]. A positive tuberculin test result for two tapirs was reported in a Swedish zoo. A culture from samples collected during the post-mortem examination of one animal exhibited growth of M. tuberculosis [9].

Tuberculosis in a Malayan tapir showed multiple white, caseous nodules measuring from 1 to $5 \mathrm{~mm}$ in diameter spread throughout the lungs [2]. The authors did not mention the manifestation of acinar processes; however, the lesions can be generalized and present in the mediastinal lymph nodes and in the liver, spleen, kidneys, ovaries, areas of the serosa, diaphragm, mesenteric lymph nodes, intestines and uterus [8].
In a case of disseminated tuberculosis caused by $M$. tuberculosis in a Malayan tapir were described multiple granulomes, some coalescent, characterized by necrotic caseous centers, in the inflammatory zone with some Langhans giant cells and a discrete fibrotic zone [2].

In the present work, the results of the anatomopathological and molecular assays confirmed the clinical suspicion of respiratory infection and established the conclusive diagnosis of pulmonary tuberculosis caused by Mycobacterium tuberculosis complex member. It is worth noting that tuberculosis is an important zoonotic disease affecting survival of the species, posing an extinction threat and also a public health concern [6].

Ethical approval. This case report was approved by the Ethics Review Board of the Universidade Federal Rural da Amazônia (approval number 23084.022512/2014-18).

Declaration of interest. The authors report no conflicts of interest. The authors alone are responsible for the content and writing of the paper.

\section{REFERENCES}

1 Brasil - Ministério da Saúde. 2008. Ministério da Saúde. Secretaria de Vigilância em Saúde. Manual Nacional de Vigilância Laboratorial da Tuberculose e Outras Micobactérias. Brasília: Ministério da Saúde, 436p.

2 de Amorim D.B., Casagrande R.A., Allevi M.M., Wouters F., De Oliveira L.G., Driemeier D., Tavares M., Ikuta C.Y., Telles E.O. \& Ferreira-Neto J.S. 2014. Mycobacterium pinnipedii in a stranded South American sea lion (Otaria byronia) in Brazil. Journal of Wildlife Diseases. 50(2): 419-422.

3 Fowler M.E. \& Miller R. E. 2008. In: Zoo and wild animal medicine: current therapy. St. Louis: Elsevier Health Sciences, 512p.

4 Freitas J.A., Ueki S.Y.M., Curcio M. \& Tury E. 2004. Identification of Mycobacterium tuberculosis in an outbreak of tuberculosis infection of a Cebus apella monkey's colony. Revista de Ciênia Agrária. (42): 165-168.

5 Kaewamatawong T., Banlunara A., Rungsipipat N., Pirarat N., Puranaveja S. \& Sommanustweechai A. 2010. Disseminated Tuberculosis in Captive Malayan Tapir (Tapirus indicus). The Thai Journal Veterinary Medicine. 40(4): 427-431.

6 Montali R.J., Mikota S.K. \& Cheng L.I. 2001. Mycobacterium tuberculosis in zoo and wildlife species. Revue Scientifique et Technique. 20(1): 291-303.

7 Morikawa V.M., Biondo A.W., Robes R.R., Barros-Filho I.R., Zimpel C.K., Lara M.C.C.S.H., Dib C.C., Gequelin L.C.F., Paploski I.A.D. \& Bonat M. 2016. Mycobacterium tuberculosis complex infection in Barbary sheep (Ammotragus lervia) at the Curitiba Zoo, southern Brazil: case report. Brazilian Journal of Veterinary Research and Animal Science. 53(3): 1-4.

8 Murakami P.S., Monego F., Ho J.L., Gibson A., Javorouski M.L., Bonat M., Lacerda O., Brockelt S.R., Biesdorf S.M., Nakatani S.M., Riediger I.N., Fuverki R.B., Biava J.S., Viera R.F., Santos A.P., Barros Filho I.R. \& Biondo A.W. 2012. Detection of RD (RIO) strain of Mycobacterium tuberculosis in tapirs (Tapirus terrestris) from a zoo in Brazil. Journal of Zoo and Wildlife Medicine. 43(4): 872-875.

9 Sternberg S., Bernodt K., Holmström A. \& Röken B. 2002. Survey of tuberculin testing in Swedish zoos. Journal of Zoo and Wildlife Medicine. 33(4): 378-380.

10 Telenti A., Marchesi F., Balz M., Bally F., Bottger E.C. \& Bodmer T. 1993. Rapid identification of mycobacteria to the species level by polymerase chain reaction and restriction enzyme analysis. Journal of Clinical Microbiology. 31(2): 175-178. 